\title{
EXPRESIONES DE LA PERSONA ADOLESCENTE SOBRE CONSUMO DE DROGAS ILÍCITAS ${ }^{1}$
}

\section{ADOLESCENT'S EXPRESSIONS ABOUT ILLICIT DRUG USE}

\author{
Ligia Murillo Castro*
}

RESUMEN

La investigación que se describe a continuación, con metodología cualitativa, a través de la técnica de dos grupos focales, se llevó a cabo en dos organizaciones no gubernamentales (ONG) de Costa Rica. Este análisis reveló las experiencias de jóvenes de ambos sexos, durante el proceso del consumo de drogas ilícitas. A la luz de sus relatos se desnuda la problemática de sus vivencias personales con respecto a las relaciones familiares, de sus amigos, educativas y con sus comunidades.

PALABRAS CLAVES: COSTA RICA * ADOLESCENTE * DROGAS ILÍCITAS * FAMILIA * AUTOESTIMA * COMUNIDAD

\section{SUMMARY}

The research described below, with qualitative methodology, through the technique of two focuses groups, was conducted in two non-governmental organizations (NGOs) in Costa Rica. This analysis revealed the experiences of young women and men during the process of consumption of illicit drugs. In light of his stories, it is bare issues of personal experiences with regard to family relations, their friends, their communities, and educative spaces.

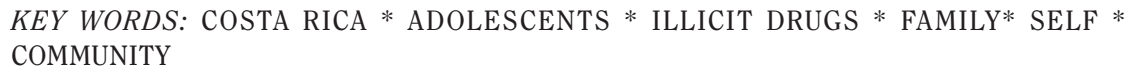

1 Esta publicación forma parte del proyecto de investigación: "El consumo de drogas en adolescentes y jóvenes en las organizaciones no gubernamentales de Costa Rica". Convenio Internacional de la Escuela de Enfermería de Ribeirao Preto de la Universidad de Sao Pablo Brasil, CICAD OEA y la
Escuela de Enfermería de la Universidad de Costa Rica.

* Escuela de Enfermería de la Universidad de Costa Rica.

ligia56@gmail.com 


\section{INTRODUCCIÓN}

El consumo de drogas ilícitas en Costa Rica entre la población de jóvenes y adolescentes constituye una problemática social muy compleja que obedece a múltiples factores, como el ambiente comunal, familiar y escolar. La situación económica sustentada en la pobreza ha conducido a la exclusión social de las familias a las que pertenecen los adolescentes, aunado a que, además, atraviesan por procesos de transformación propios del desarrollo de los jóvenes en esta etapa de la vida. Las diversas dificultades a las que, cotidianamente, se enfrentan estos adolescentes generan vulnerabilidad $y$, por lo tanto, una mayor probabilidad del consumo de drogas ilícitas. La facilidad con que cuenta la juventud para la adquisición de los diversos tipos de alcaloides - tabaco, marihuana, cocaína, crack - en el país, que en su mayoría son suministrados por los adultos o por los grupos de pares; en el caso de estos últimos simbolizan para los jóvenes el único apoyo, debido a los diversas dificultades que tienen con sus familias $y$ ante la imposibilidad de los padres para afrontar la adicción de sus hijos. Y, por supuesto, también influye el hecho de que la drogadicción se gesta en su propio contexto social. El fenómeno de las drogas es una problemática que requiere analizarse desde las políticas de las instituciones del Estado Costarricense.

Por lo tanto las disposiciones sobre niñez y adolescencia creadas por el Patronato Nacional de la Infancia (PANI) y el Consejo Nacional de la Niñez y Adolescencia (2005) establecen que las políticas económicas y sociales dictadas por el Estado en relación con oportunidades de empleo, producción y acceso a la vivienda digna, deben encaminarse hacia las metas del desarrollo; así, la normativa procura que todas las familias, especialmente aquellas que se encuentran en situaciones de pobreza, tengan acceso al cuidado de los niños, niñas y adolescentes con el propósito fundamental de que logren una mejor calidad de vida.

Sin embargo, la realidad en Costa Rica, a pesar de las pautas establecidas para este grupo poblacional de niñez y adolescencia, nos devela que no todos los núcleos familiares logran obtener las mencionadas oportunidades de desarrollo para sus miembros. Así, lo reflejan los jóvenes entrevistados para el presente estudio, por cuanto el trasiego y el consumo de drogas ilícitas no solo forman parte de su vida cotidiana, sino también constituyen una entrada para su propia subsistencia, un elemento clave para su medio ambiente familiar y comunitario.

Así lo revela el estudio de Ugalde et ál (2003 citado por Bejarano, 2005), en el sector educativo, la problemática por el uso de drogas entre los muchachos con edades comprendidas entre los 15 y 17 años muestra niveles de prevalencia superiores en el consumo del último mes en un 33,2 para el alcohol, 21,5 para tabaco, 3,3 para marihuana $y$ un 0,9 por ciento para cocaína y crack. No obstante, estos jóvenes del estudio refieren que se inician en la adicción a muy temprana edad; empiezan con cigarrillos y alcohol para luego pasar a drogas más complejas como la marihuana y el crack.

La investigación cualitativa, que se efectúa en el periodo comprendido de julio a octubre de 2008, permitió conocer acerca del consumo de las drogas ilícitas a través de la técnica del grupo focal, las vivencias personales de 16 adolescentes mujeres y hombres que están institucionalizados en las organizaciones no gubernamentales del país para rehabilitarse de la adición a las drogas.

\section{OBJETIVOS DEL ESTUDIO}

OBJETIVO

Analizar los factores del entorno en el transcurso del consumo de las drogas ilícitas en las personas adolescentes institucionalizadas en las organizaciones no gubernamentales de Costa Rica.

\section{OBJETIVOS ESPECÍFICOS}

1. Identificar los factores propios de las adolescentes $y$ los adolescentes en el espacio del consumo de drogas ilícitas

2. Analizar la dinámica familiar de la persona adolescente drogodependiente

3. Determinar la influencia de la escuela o colegio y la comunidad en el lapso del consumo de drogas de los adolescentes 


\section{METODOLOGÍA}

\subsection{ACERCAMIENTO METODOLÓGICO}

Este estudio se basó en la investigación cualitativa que es, "el procedimiento metodológico que utiliza palabras, textos, discursos, dibujos, gráficos e imágenes para comprender la vida social por medio de significados $y$ desde una perspectiva holística, pues se trata de entender el conjunto de cualidades interrelacionadas que caracterizan a un determinado fenómeno" (Mejía, 2004: 278). De esta manera, la investigación analiza el entorno en el transcurso del consumo de las drogas ilícitas, expresado desde las propias voces de los adolescentes $y$ las adolescentes, desde sus significados $y$ sus vivencias para explicar el fenómeno social de la drogodependencia en un grupo poblacional específico, constituido por jóvenes.

\subsection{EL ENCUENTRO CON LOS JÓVENES DE LA INVESTIGACIÓN}

Los sujetos del presente análisis están constituidos por dos organizaciones no gubernamentales de Costa Rica; dichas entidades nos concedieron el permiso para llevar a cabo la investigación. Estas instituciones albergan adolescentes para la rehabilitación del proceso de la drogadicción. La población escogida proviene de dos Hogares Crea: Hogar Crea de Heredia, en Santa Lucía, que trabaja con hombres adolescentes, y Hogar Crea de Cartago, que proporciona tratamiento a mujeres adolescentes. Se seleccionó a ocho adolescentes en cada uno de los centros de atención y en ambos se empleó la técnica de grupo focal, que tiene como propósito fundamental en la investigación social, "lograr una información asociada a conocimientos, actitudes, sentimientos, creencias y experiencias que no serían posible de obtener, con suficiente profundidad" (Aigneren, 2009: 2). La escogencia de este grupo de adolescentes para que formaran parte de la investigación se efectuó de manera intencional. Por criterios de inclusión asumidos fueron la pertenencia a un programa de rehabilitación para superar el consumo de drogas ilícitas, en las dos institu- ciones mencionadas. Además, de que estuvieran en una fase de rehabilitación avanzada, para evitar la deserción de los participantes durante el estudio y que se comprometieran a una aportación voluntaria mediante la firma de un consentimiento por parte de cada adolescente.

Como técnica de investigación cualitativa, se trabajó con dos grupos focales compuestos por hombres $y$ mujeres. El proceso se realizó mediante encuentros separados para compartir cada percepción en discusiones guiadas por la investigadora, acerca de su proceso de drogadicción. Además, de acuerdo con las categorías de análisis considerando sus percepciones, el entorno de la familia, la escuela y la comunidad, según agenda diaria de encuentros que fue confeccionada para tal fin. También se consultó a informantes clave, los cuidadores de los adolescentes en ambas ONG, así como a miembros del personal administrativo; este se compone de dos psicólogas y seis hombres que se desempeñan en puestos de supervisión, dirección y sudirección de las dos instituciones $y$ que han experimentado un proceso de drogadicción y recuperación. Se aplicó un instrumento abierto con interrogantes pertenecientes a las mismas categorías de abordaje a través de una guía para los grupos focales.

\subsection{ACCESO AL CAMPO CON EL GRUPO DE ADOLESCENTES}

Cada sección fue grabada, con el consentimiento de los jóvenes, durante cada uno de los encuentros con los dos grupos focales. Se recolectó la información paulatinamente de acuerdo con los objetivos planeados y al nivel conversacional que se generó en el seno de cada grupo; luego fue necesario diseñar una unidad de análisis programada para sistematizar la información. Con base en las mismas categorías contempladas en los instrumentos, se trataron las categorías del ambiente que giran en torno a su proceso de vida de las personas adolescentes, $y$ a los factores que incidieron en el transcurso de la adicción a las drogas ilícitas, así como su propia percepción sobre el entorno de la familia, la escuela y la comunidad. 
Una vez que se obtuvo el punto de saturación, se procedió al análisis de la información; de igual manera, los datos se trataron para el grupo de los informantes clave o cuidadores. Con el objeto de documentar los relatos de los adolescentes e informantes se empleó "h" para el grupo de los hombres; una "m", para las mujeres, y una "ic", para los informantes clave, y se agregó para cada participante de cada grupo una numeración del 1 al 8 .

\section{REFLEXIONES DESDE LOS JÓVENES}

Una vez concluida la aplicación de los instrumentos a los actores participantes en la investigación, es decir, a las personas adolescentes, así como a los informantes clave o cuidadores, se procedió a elaborar el análisis de la información, que a continuación se delimita en forma individual en representación de relato para respetar lo que expresó cada adolescente, ya que la realidad de cada uno es diferente aunque la problemática del consumo de las drogas tiene algunas similitudes como grupo social.

\subsection{PERSONALIZACIÓN DE LOS ADOLESCENTES}

Como se puede visualizar en la tabla 1 , las edades del grupo estudiado oscilan entre los 14 y 18 años, que son los parámetros establecidos para estos muchachos, vistos como adolescentes según el Patronato Nacional de la

TABLA 1

HOGARES CREA: ADOLESCENTES DE PROVINCIAS DE CARTAGO Y HEREDIA. COSTA RICA, CENTROAMÉRICA (SEGÙN EDAD Y SEXO)

\begin{tabular}{cccc}
\hline EDAD & MASCULINO & FEMENINO & TOTAL \\
\hline 14 & 2 & 4 & 6 \\
15 & 2 & 2 & 4 \\
16 & 0 & 1 & 1 \\
17 & 4 & 0 & 4 \\
18 & 0 & 1 & 1 \\
\hline TOTAL & 8 & 8 & 16 \\
\hline
\end{tabular}

Infancia (2005) en nuestro país y como menores de edad. No obstante, existe una predominancia en el consumo de drogas ilícitas por sexo según se observa en las mujeres a los 14 años. Sin embargo, es importante destacar, con base en la clasificación que hace el autor Dulanto (2000), se consideran como adolescencia temprana a las mujeres de 14 años, $y$ los hombres a los 17 años como adolescentes medios. Asimismo según el autor Solum (1999), la adolescencia y la juventud están contempladas entre las edades de 10 a 24 años, concurrente en este grupo estudiado, periodo que constituye un proceso evolutivo si se consideran las dimensiones biológicas, psicológicas y sociales, $y$ se reflexiona en un entorno cambiante que acontece en lo histórico y lo social del contexto de cada adolescente.

\subsection{LA AUTOESTIMA DE LAS PERSONAS ADOLESCENTES QUE CONSUMEN DROGAS ILÍCITAS}

Dentro del espacio personal de la adolescencia se encuentra la autoestima, la cual va estar determinada por la concepción que cada joven tenga sobre sí mismo(a) y las percepciones que los demás tengan de él o ella. Según Dulanto (2000), la autoestima en la persona adolescente, es aquella capacidad de apreciarse por el mismo adolescente, es el aprecio propio que uno tiene. De forma semejante, este autor afirma que la autoestima en el individuo joven es la manera que posee de examinarse correspondiente a la forma, que lo hace sentirse merecedor, apreciable, ante él o ella misma y ante los demás.

La autoestima se halla estrechamente vinculada con aquellas fortalezas, las prácticas de vida que se forman $y$ se manifiestan en las personas jóvenes; así, lo define la autora en la siguiente cita:

La autoestima es el conjunto de experiencias subjetivas $y$ de prácticas de vida que cada persona experimenta $y$ realiza sobre sí misma. En la dimensión subjetiva intelectual, la autoestima está conformada por los pensamientos, los conocimientos, las intuiciones, las dudas, las elucubraciones 
y las creencias acerca de una misma, pero también por las interpretaciones que elaboramos sobre lo que nos sucede, lo que nos pasa y lo que hacemos que suceda. Es una conciencia del Yo en el mundo y, por ende, es también una visión del mundo $y$ de la vida. Y en la dimensión subjetiva afectiva, la autoestima contiene las emociones, los afectos $y$ los deseos fundamentales sentidos sobre una misma sobre la propia historia, los acontecimientos que nos marcan, las experiencias vividas y también las fantaseadas, imaginadas y soñadas (Lagarde, 2000: 9).

Los adolescentes, en esta investigación, expresan sobre sí mismos aspectos que forman parte de su experiencia de vida que les permite descubrirse, remirarse; en ocasiones expresan sentimientos de minusvalía:

- (...) Sentía soledad y miedo, muchos vacíos; sufría de depresiones; no valía nada. (m.1)

- (...) Sentía que no le importaba a nadie. (m.3)

- (...) Busqué mis amigos; me sentía solo, baja autoestima, baja confianza; evadía los problemas sin drogas. Ya con drogas fue peor. (h.1)

—(...) Me gusta ser honesta y sincera. (m.5)

—...) Soy una persona muy especial. (m.7)

- (...) Me cuesta perdonar; me echo la culpa de todo. Me gusta hacer cosas nuevas. No me gustan las rutinas. (m.9)

- (...) Yo tenía autonomía para tomar las decisiones; trabajaba y toda la plata que me ganaba era para consumir droga. (h.4)

Equivocarse, cometer errores al consumir las drogas es una experiencia que se origina en su entorno, en la relación con sus amigos y la familia; así, lo enuncian ellos y ellas:
-(...) Sentía odio, rencor, vacíos. Yo, entonces, empecé a buscar gente negativa, por los problemas de mi familia y porque mis papás se separaron. (m.4)

- (...) Para aceptar que tenemos un problema con las drogas, se nos hace muy difícil. Nos falta confianza para admitir, sino hasta que cometemos delitos y caemos en algo muy grave. (h.8)

Las opiniones vertidas por los adolescentes reflejan sentimientos muy negativos hacia sí mismos y para con su entorno. La vida cotidiana, experimentada como un inmenso remolino de conflictos, los hace sentir mucha frustración, desesperanza e indignación, emociones que durante las sesiones grupales se emitieron a través del testimonio de cada muchacho o muchacha.

Es significativo mencionar la adolescencia como parte del ciclo de vida, en el que los jóvenes tienen la influencia de los factores protectores, constituye un enfoque del que se viene discutiendo hace algún tiempo en el ámbito de la salud pública. (Según Rutter 1992 y Serrano 1995 citado por Rojas Milton S/F), se denominan factores protectores a las condiciones $y$ atributos que facilitan el logro de la salud integral del adolescente $y$ las relaciones con la calidad de vida en el nivel individual y grupal.

Algunos de los adolescentes se fortalecen en el transcurso de su vida, pero otros no alcanzan este proceso por diversas circunstancias en su propia historia de vida $y$, en consecuencia, caen en procesos de riesgo de consumir sustancias que conllevan vivencias diferentes en las personas $y$ en sus propios acontecimientos. Los factores protectores denominados como acontecimientos y particularidades propias, especiales, que proveen el beneficio de una salud integral para que los niños y adolescentes mejoren su calidad de vida tanto en escala individual como grupal.

Diferentes estudios en los grupos de adolescentes muestran, según relata Rojas Milton (s/f), el consumo de sustancias psicoactivas, lo cual provoca que los muchachos y las muchachas busquen resolver sus problemas a través 
de la adquisición de las drogas; esta se da con mucha facilidad en el medio ambiente social en el que habitan. Así, lo expresan algunos jóvenes:

- (...) Mi mamá alcohólica y mi padrastro también: él agredía a mi mamá. Me sentía muy sola. Busqué, entonces, personas negativas. Me sentía menos que los demás. (m.5)

- (...) Inicié tomando licor hasta emborracharme. Cuando ya no me hacía efecto, empecé con las drogas. (h.3)

- (...) Inicié fumando; luego decidí tomar licor $y$ después pase a la marihuana. Y, cuando ya sentí que necesitaba un efecto más grande, probé todas las demás drogas como el crack. (h.4)

- (...) Al principio me sentía sola; por eso busqué amigos. Con ellos me sentía bien; con la droga me sentía que no dependía de nadie. (m.2)

La adolescencia es considerada por algunos autores en el mundo de los adultos, como un proceso crítico de paso, por cuanto le permite a los jóvenes entrar en una etapa que les provoca alguna crisis, debido a todo el proceso de transformación tanto en el ámbito físico, emocional, como espiritual, que tienen que emprender. El desarrollo del ser humano por la senda de la vida es el camino que recorre un niño o una niña para formar parte de un nuevo ser, con la experimentación de un cuerpo diferente y también algunas experiencias que antes no conocía. Igualmente, el autor Dulanto, define la adolescencia de la siguiente manera:

Un proceso de tránsito determinado por varios factores: La vida en familia, la experiencia escolar, el marco cultural que ha estructurado el mundo social para la puesta en práctica de normas y limites que forman la base educativa $y$ explican la experiencia de la vida comunitaria y las condiciones económicas y políticas que imperan en la generación que pasa por la experiencia de ser adolescente, momento sociohistórico (Dulanto, 2000: 143).

La vida de la persona adolescente socialmente representa a un grupo, que ha sido considerado como diferente, afrontando las críticas $y$ dificultades del medio social en que viven. Los adolescentes deben considerarse como parte del producto de la época, de la cultura de donde provienen, de su propia historia de vida y de su entorno familiar y comunal.

Para algunos adolescentes, su sentir muy propio y la forma en que miran su vida se expresan, donde se consideran omnipotentes de la siguiente manera:

- Me gusta vacilar; tengo poca confianza; no me gusta expresar. Me gusta experimentar; me gusta la velocidad, como andar en moto; me gustan los deportes extremos. (m.4)

- Nosotros somos personas que tomamos decisiones pero mal encaminadas; al final, cuando nos sentimos perdidos es cuando tenemos una toma de decisiones, pero para buscar ayuda; por algo llegamos a un lugar como este para rehabilitarnos. (h.8)

- Me gusta la playa; me gusta solfear. (m.2)

- Me gusta hacer dibujos; he hecho algunas cosas con barro, como pasatiempo favorito. (m.6)

Los sentimientos expresados por estos muchachos, aun considerando la situación de la drogodependencia en la que están inmersos, forman parte de las emociones, impresiones y mensajes de un adolescente subordinado a un proceso adaptativo frente a sí mismo y frente a los demás, considerando los desafíos a los que se enfrentan como grupo, con conductas retadoras como las que fueron formuladas por los jóvenes.

Uno de los aspectos importantes en la adolescencia es la identidad como proceso. Resulta trascendental, por cuanto empieza a gestarse, a definirse en la experiencia de lo 
central y vital de la persona, Amara (2000). Esta fase constituye un periodo del sentido creciente del yo, en el cual él o ella tratan de ajustarse al medio donde se desenvuelven como personas, con sus propias vicisitudes. Según Erickson (1994), citado por Amara (2000), la identidad es la perspectiva del estado mental del yo; es un espacio donde se siente más profundo e intensamente activo $y$ vivo.

El joven llega, por su propio camino y a su propia manera, a este crecimiento y maduración, que se forja afrontando cotidianamente a raíz de los problemas que le rodean. Es normal que experimente una dificultad y que muestre un cierto grado de desorganización y confusión, ya que debe establecer un logro de identidad; esto implica definir aspectos clave de sí mismo $y$ de su relación con el ambiente y su entorno cultural; al respecto, la autora define la identidad en la adolescencia así:

La identidad es considerada como un desarrollo en la interface entre lo social $y$ el sí mismo encarnado en la persona, como la autoría del sí mismo. Esta autoría es la capacidad de hablar sobre sí mismo y establecer un diálogo interno en el que se organizan las distintas voces que expresan los discursos sociales y se genera una voz propia (Hernández, 2006: 462).

En este grupo social, es decir, los adolescentes que consumen drogas, los hombres, a través de sus relatos, describieron una identidad negativa, citada por el mismo autor Amara (2000). El adolescente es transformado en una nueva persona, que lo inclina a cometer actos amorales, prohibidos o de riesgo, que lo convierte en un extraño para los miembros de su propia familia y los amigos; asimismo, lo confronta con su yo ideal, lo que ocasiona como consecuencia un choque con él mismo, en este caso particular reconocen que tienen un problema con la adicción a las drogas, que necesitan ayuda para resolverlo o, en otras palabras, toman conciencia de que no lo pueden hacer solos, y es el momento justo en que se acercan a una institución, como las organizaciones no gubernamentales, para iniciar un proceso de rehabilitación.
En las expresiones anteriores, el grupo de mujeres se describen, en la identidad, con más atributos positivos, henchidas de fuerza, de esperanza; nos revelan cómo, a pesar de la adversidad, encarnan sentimientos muy propios de la adolescencia, como la pasión por experimentar nuevas experiencias.

Existen diversos aspectos que giran en torno a la adolescencia; sin embargo, se debe establecer la diferencia y respetar las individualidades. El análisis de cada realidad marca la diferencia para conceptualizar a un grupo poblacional que es proclive a los cambios y que, al mismo tiempo, está marcado por el impacto de cada época en particular; en la actualidad se halla influenciado por la globalización de los mercados, el consumismo, el uso de la tecnología cada vez más sofisticada, el mundo de la moda, la música; estos dos últimos simbolizan para nuestros adolescentes un modelo a seguir, generalmente imitado por muchos jóvenes, y que además conlleva mensajes ocultos, como el uso de las sustancias lícitas e ilícitas, para sentir creatividad y movimiento. Al respecto el autor Martín menciona sobre el papel que juegan los medios de comunicación lo siguiente:

Numerosos análisis han demostrado a los largo de las últimas décadas la influencia que los medios audiovisuales tienen en los espectadores, en especial en los menores de edad. Por eso podemos concluir que la televisión es uno de los principales vehículos de transmisión de valores en nuestra sociedad. Las madres, padres y educadores están no solo en desventaja, sino que parecen están perdiendo capacidad de influencia en detrimento de los medios de comunicación audiovisual: (Martín, Javier, <www.unad.org/>)

Esta forma de transmitir una comunicación distorsionada le encanta al adolescente, le llama la atención y lo conduce hacia caminos desviados, donde afrontará múltiples riesgos, que luego van a desembocar en consecuencias muy nefastas. Así, lo sostienen en los siguientes relatos:

- (...) Necesitaba un efecto más grande; probé todas las demás drogas, entre ellas, como el crack. (m.6) 
-(...) Me ubicaba en un cajero. Asaltábamos a las personas colocándoles un puñal; les quitábamos el dinero. (h.2)

- (...) Consumía licor con marihuana; y me agarró el palidazo: sudor, vómitos. Se pone todo oscuro; se oye como un eco. (m.8)

- (...) El estilo de vida de nosotros los drogadictos es el consumo. Después, no hay nada; ni siquiera dormimos ni comemos. Solo pasamos drogados. (h.5)

- (...) Me robaba cosas de mi casa para venderlas por droga. (m.3)

- (...) Empecé a robar en mi propia casa: el televisor, la grabadora, pero luego empecé asaltar casas y me robaba lo que encontrara más fácil, pero me llegan a detener porque me vieron y me denunciaron a la policía. Después me trajeron aquí a CREA. (h.1)

Las mujeres adolescentes como se puede observar en los anteriores relatos son más expresivas que los hombres. Existen sentimientos en ambos sexos de minusvalía, de baja autoestima, conflictos familiares, soledad $y$ desesperanza; se convierten en delincuentes para adquirir las drogas porque representan para los muchachos una salida equivocada tendiente a aminorar las situaciones conflictivas que desafían. Según un estudio de IAFA (2006) los problemas de la salud mental detectados en adolescentes, fueron: la ansiedad, depresión y desórdenes cognitivos, así como los sentimientos de desesperanza y tristeza constituyen el rango más sobresaliente.

La edad promedio de este conjunto de jóvenes se ubica en la adolescencia media, la cual, según lo manifiesta Dulanto (2000), es la etapa de la vida en la que él o ella tiene los sentimientos de experimentación y omnipotencia. Esto es lo que favorece, en ese grupo poblacional, la toma de decisiones equivocadas para el inicio del consumo de drogas lícitas; primero, como un mecanismo para disminuir sus emociones negativas $y$, segundo, al buscar nuevas experiencias $y$ de mayores sensaciones inicia el consumo de drogas ilícitas; así lo han manifestado ellos mismos. De manera que la adolescencia, según refiere Díaz J. (2006), también ha sido considerada como una etapa que se identifica con problemas de diversa índole: emocionales (duelos y crisis), conductuales (estados cambiantes, rebeldía) y sociales (aislamiento, transgresión a las normas). Si bien esas manifestaciones son reales, no constituyen parámetros absolutos para definir a todos los adolescentes en todos los contextos. Así, lo expresan los adolescentes que han entrado al mundo de las drogas:

- (...) Empecé fumando cigarrillo a los 11 años y tomando licor; luego pasé al consumo de marihuana. (m.5)

- (...) Empecé fumando marihuana porque siempre veía a mi abuelito fumar; de ahí aprendí. (m.1)

- (...) Inicié tomando licor hasta emborracharme. Cuando ya no me hacía efecto, empecé con las drogas. (m.1)

En esta fase, el adolescente no tiene conciencia de lo que está adquiriendo realmente con el consumo de las drogas ilícitas. La experimentación, una vez que se inicia, no puede parar. Según este grupo estudiado de hombres $y$ mujeres adolescentes, el joven es agobiado por la conducta adictiva de las mismas drogas $y$ por el deseo de buscar más efecto en este proceso para abrumar sus penas. Según un estudio de Mendizábal J. y Anzures B. (1999), la interacción entre el adolescente y sus padres frecuentemente se caracteriza por una pobre comunicación y una expresión afectiva negati$v a$, que derivan en un manejo inadecuado de los recursos para el control de los límites. De igual manera, en esta investigación, los sentimientos de inestabilidad de los jóvenes al principio del consumo son siempre de crisis, de conflicto más evidente dentro de la relación familiar, que buscan el alivio en el consumo inicial de drogas.

De esta manera, podemos resaltar que los factores protectores que según (Rutter, 1992; 
Kandell, 1978; citado por Rojas Milton s/f), son la autoestima, el proyecto de vida, escala de valores, tales como: el amor, la verdad, la libertad, el respeto, la religiosidad, la moral; también se incluyen los microambientes como los familiares, escolares, laborales, deportivos $y$ recreativos, la responsabilidad en la salud reproductiva, el uso de redes de apoyo. Todos estos elementos protectores deben estar en las personas en cualquier ciclo de la vida; sin embargo, simbolizan, en los jóvenes de la sociedad actual, una convivencia más sana con los demás seres humanos y su entorno, si tienen la posibilidad de contar con ellos.

El reconocimiento de estos componentes positivos permite disminuir la vulnerabilidad $y$ el riesgo de consumir tanto drogas lícitas como ilícitas o alguna otra conducta de riesgo; en consecuencia, debe evidenciarse cada día más el trabajo de los profesionales en las ciencias de la salud mediante el impulso y desarrollo de los programas preventivos $y$ de fortalecimiento. Un plan de esta índole tendrá como cometido combatir los problemas inmediatos de la mencionada población; la idea debe orientarse hacia a los diversos grupos sociales como los padres de familia, los maestros, líderes religiosos, líderes juveniles, personal que atiende a la niñez y a la adolescencia en diversas organizaciones nacionales, con la finalidad de mejorar la calidad de vida de la niñez y la adolescencia costarricenses. No obstante, resulta necesario enfatizar en que es función del Estado costarricense proporcionar a las familias de los niños, niñas $y$ adolescentes, en el contexto nacional, mejores condiciones en la dimensión social y ambiental, tales como oportunidades de empleo, salarios justos, vivienda digna, mejor acceso a la educación y a los servicios de salud. Estos factores permitirán mejorar la calidad de vida de nuestros muchachos $y$ nuestras muchachas.

\subsection{LA FAMILIA Y EL ADOLESCENTE EN LA DROGODEPENDENCIA}

La familia simboliza un medio, según Minuchin S., Fishman H., citado por Mendizábal J. y Anzures B. (1999), que permite la crianza de los hijos y representa la influencia más temprana $y$ duradera para el proceso de socialización; además, activa la comunicación entre sus integrantes y la sociedad.

Los acontecimientos que ocurren en el núcleo familiar, como lo afirma Horkheimer (2001), forman parte de la persona desde el periodo de la niñez y tienen una función clave, que consiste en permitir el desarrollo de sus capacidades.

Sin embargo, el ambiente familiar en la actualidad no siempre es armonioso debido a todos los embates que enfrenta la sociedad actual, regida por un sistema capitalista que involucra componentes como los procesos económicos, las diversas formas de producción, el trabajo, el consumismo, el desempleo, la pobre$z a$, la violencia, la convivencia en pareja, entre otros. De manera tal que en el acontecer de nuestra sociedad, los grupos sociales reaccionan $y$ esto se debe a diversos dominios globales de la sociedad en su conjunto, que funcionan diferente en cada clase social, según Hidalgo y Gutiérrez (2007), el ambiente de los adolescentes ha cambiado y cada vez se ven sometidos a más dificultades y a nuevos riesgos. La duración de este proceso en el tiempo y en el espacio social ocasiona una influencia en las instituciones estables como la familia, la iglesia, $y$ otras organizaciones, según Horkheimer (2001), lo cual desemboca en que se transformen, en que los cambios surjan paulatinamente. Así, lo enuncia el autor, al referirse a la familia, en la siguiente cita:

Representa una de las formas sociales que, como elementos del edificio cultural presente, ejercen sus funciones, en sí necesarias, de manera cada vez más deficiente, a causa de las contradicciones $y$ crisis que se van agudizando, sin que, no obstante, puedan ser cambiadas fuera del contexto general de la sociedad. (Horkheimer, 2001: 207).

La familia es una construcción social; se ha descrito como un modelo institucionalizado bajo las normas establecidas, tareas y funciones que deben ejecutar los padres como seres hegemónicos, con un comportamiento sistémico entre sus miembros, a través de la figura de 
autoridad. No obstante, en el acontecer social actual, la dinámica familiar ha experimentado transformaciones $y$ ha generado conflictos que, en ocasiones, no pueden ser zanjados por sus integrantes.

Uno de los aspectos enunciados por los adolescentes, cuando se inician en el consumo de las drogas, son las dificultades que surgen en la relación cotidiana familiar.

A continuación, esta realidad se expone por medio de las propias voces de los adolescentes y las adolescentes:

- (...) La relación con la familia es muy mala. Me sentí despreciada por la familia; conflictos con los hermanos y con los padres. (m.4)

-(...) Habían muchos problemas en mi familia; mi mamá me agredió mucho.

Me golpeaba. (m.6)

-(...) Mi papá tomaba licor enfrente de mí y mi hermana; yo me enojaba y le botaba las botellas. Empecé a consumir licor para huir de los problemas y para experimentar qué se sentía (m.7)

-(...) Las relaciones muy conflictivas entre mis padres $y$ entre toda la familia; siempre un ambiente rodeado de muchos problemas. (h.3)

Los informantes clave opinan que una de las mayores dificultades de los adolescentes adictos a las sustancias ilícitas está en la relación familiar, aspecto que ellos mismos expresaron así:

- (...) La familia es disfuncional debido a que los padres, la mayoría del tiempo, han socavado la autoestima de los adolescentes. (i.c.8)

- (...) En cuanto a los adolescentes que consumen drogas, la relación que tienen con sus padres es distante, fría, rebelde. (i.c.4)

La familia es la unidad social históricamente reconocida a través de las distintas épocas.
En la etapa de la adolescencia representa para los muchachos un factor protector porque los padres asumen el papel de brindar y satisfacer las necesidades básicas, así como favorecer el desarrollo psicosocial de la joven y del joven mediante un vínculo afectivo, que une a sus integrantes en el medio ambiente familiar. Sin embargo, existen mecanismos de crisis familiar que permiten a la familia la búsqueda del equilibrio y la homeostasis. La familia ha sido definida por el modelo de sistemas, según Levetón (1987), como un sistema vivo, cuyos miembros interactúan entre sí y cuando una situación particular afecta a uno de ellos, tiene influencia sobre el resto de los integrantes. La crisis de disfuncionalidad, provocada por el tráfico y el consumo de la droga, es percibida por los mismos adolescentes como se transcribe a continuación:

-(...) Mi papá me encontró probando fumar, mas me obligó a fumarme un cigarro completo para que probará; luego él y yo fumamos y tomábamos licor juntos. (m.1)

-(...) Mi papá y mi mamá vendían droga; siempre habían muchos conflictos en el hogar. Desde niño pasé viendo la policía entrar y salir de mi casa. Toda la familia es narcotraficante de la droga. (h.1)

La familia se constituye, en cada país y en cada sociedad, dentro del marco de su propia dinámica. Según Fishman (1989), el núcleo familiar se ha convertido en una institución inestable que ha pasado de un modelo de familia vertical tradicional a un sistema horizontal, constituido por los padres, amigos $y$ auxiliares, se produce como consecuencia de que el adolescente recurre a buscar más apoyo en sus pares $y$ hermanos que en sus propios padres. Otro aspecto que menciona el mismo autor, con respecto a las familias en el presente, es que los progenitores como figuras parentales son ineficaces al ejercer su conducción en el hogar porque en la mayoría de ocasiones están en desacuerdo crónico, los roles no están establecidos, existe ausencia de límites y de una autoridad, por lo tanto, se produce una división entre los miembros del hogar. 
Este grupo estudiado de las familias de los adolescentes está constituido por mujeres jefas de hogar, a quienes sus compañeros han abandonado a su suerte $y$, en consecuencia, los padres no han asumido la responsabilidad paterna de los hijos; como resultado, ellas han tenido que asumir trabajos informales en los que obtienen un recurso económico muy limitado para el sustento de sus hijos; de manera que la mayoría de los muchachos y muchachas son de origen pobre, en otras palabras, de recursos económicos muy escasos. Solamente una adolescente refirió que están presentes los dos padres en el hogar, pero las relaciones resultan sumamente conflictivas, situación que ha sido expresada por los jóvenes y cuidadores anteriormente.

Otro componente importante dentro del círculo familiar de este grupo poblacional es que por lo menos uno de los miembros se dedica al consumo y trasiego de drogas ilícitas; por lo tanto, los adolescentes se ven inmersos en este proceso desde edades muy tempranas, por cuanto son los mismos padres, hermanos o familiares quienes los introducen en este flagelo humano. La pobreza, el tráfico y consumo de drogas en los núcleos familiares trae aparejados conflictos muy funestos $y$ la disfuncionalidad familiar. Tal panorama se refleja en los diversos tipos de abuso que sufren estos adolescentes, según lo manifiestan:

\section{- (...) Empecé a tener relaciones con un narco;} pasaba en los moteles drogada. (m.4)

- (...) Una mujer por mi casa, mayor que yo, sabía que consumía drogas; entonces, me proponía tener relaciones sexuales con ella. Luego me pagaba y yo gastaba el dinero en drogas. (h.3)

- (...) Tuve las relaciones en el monte; nunca me importó; siempre estuve drogada; no sentí nada. Me prostituía por la droga. Tuve diferentes hombres: sus edades estaban desde los 14 años hasta los 40 años. (m.8)

- (...) Aquí hay muchos que se han prostituido por las drogas, pero no lo quieren decir.
Yo, sin embargo, sí. Una mujer me pagaba dinero para que tuviera relaciones sexuales con ella. (h.7)

- (...) Yo me junté con un mes con un muchacho; luego, con otro. Me junté como tres veces con diferentes hombres. (m.1)

- (...) En la adicción, me involucré con una mujer de 33 años, viuda. Ella vive en el barrio a cinco casas de mi vivienda; ella me echaba el cuento; yo tenía relaciones sexuales con ella y luego me daba plata para consumir. (h.5)

Como se infiere de sus declaraciones, uno de los inconvenientes que afrontan estas muchachas y estos muchachos son los diversos tipos de abuso, como el físico desde los mismos padres y el abuso sexual de los adultos hacia los y las jóvenes, según las expresiones de ellos mismos, lo que contribuye a forjar un medio para seguir consumiendo drogas a fin de mantener la propia supervivencia del adolescente $y$ el funcionamiento de un círculo del cual no pueden salir.

El abuso sexual en los menores de edad se define como las relaciones carnales "que mantiene una persona menor de 18 años con un adulto o con otro menor de edad, para las que no está preparado evolutivamente $y$ en las cuales se establece una relación de sometimiento, poder y autoridad sobre la víctima". (Arenas y Villalobos, 2008: 9). De esta forma resultan ser los adultos quienes atropellan a los jóvenes a través de las distintas formas de abuso; ejercen su poder y su fuerza para aprovecharse del propio proceso de adicción a las drogas. En consecuencia, se torna más difícil el período de recuperación de los adictos. Así, se originan derivaciones en el daño físico, moral, psicológico que soporta un adolescente con cualquier tipo de abuso, circunstancias que evidencian, según Brindis (1997), citado por Hesse M., Vanderplasschen W., Rapp R. C., Broekaert E., Fridell (2008), lo multifacético y complejo de los problemas de abuso de drogas, pues los programas de tratamiento que abordan la crisis del consumo vienen a ser insuficientes. 


\subsection{LOS ADOLESCENTES Y SUS AMIGOS}

Los amigos representan para los jóvenes un aspecto relevante en la etapa de la adolescencia media, entre los 15 y 17 años, es decir, precisamente el grupo etario al que pertenece el conjunto de adolescentes de esta investigación. $\mathrm{El}$ apoyo incondicional de los pares forma parte de una subcultura creada por ellos mismos, de acuerdo con sus valores, principios, normas, forma de pensar, de vestir, gustos y preferencias que son compartidos en común. Tienen, además, su propia forma de comunicarse, sus códigos y símbolos fundidos en un solo crisol: el propio grupo social.

Los mismos procesos de cambio a los que se ve enfrentado el adolescente derivan en que su identidad se determine por la concepción de sí mismo, a través de su propio yo. Y, además, descubre al mismo tiempo la necesidad de interacción social; pasa de un extremo a otro, en especial en la relación con los demás, en una nueva búsqueda de la perspectiva del mundo. Este camino lo emprende para resolver las situaciones conflictivas que lo abaten.

En este proceso de definición de identidad, de búsqueda y de cambio, los amigos constituyen un factor clave para los adolescentes, por cuanto en la escala de valores del muchacho o la muchacha viene a ser tan importantes como su propia familia. Según Castellano (2005), en la adolescencia y juventud, el papel de los amigos es fundamental en el desarrollo evolutivo, hasta el punto de que pueden considerarse como su segunda familia. En el complejo proceso de integración, la amistad es una relación social; surge a través del espíritu aventurero $y$ de interdependencia del joven.

En particular en el proceso de consumo de drogas ilícitas y de la rehabilitación para aquellos jóvenes que desafían este período, los amigos personifican la experimentación al consumo de drogas ilegales, el cual no surge como un comportamiento ajeno o exclusivo de la juventud, sino más bien como una actitud que afecta al común de la sociedad.

La relación de amistad de las y los adolescentes, con sus amigos o pares la externan de la siguiente manera:
-(...) Mis amigos me veían tan golpeada que me ofrecían la droga; inicié a los 12 años. (m.5)

-(...) Mis amigos influyeron mucho; quería ser aceptada por ellos y, para eso, tenía que consumir. (m.1)

-(...) Los amigos influyen porque uno está muy confundido. (h.6)

-(...) Los amigos negativos consumen la droga y influyen mucho para que nosotros la consumamos también; si no consumes, no eres parte de su grupo. (h.4)

-(...) Algunos de mis amigos eran buenos, pero yo les fallé; les hice mucho daño. Al final, me quedé sin ellos. La droga te consume poco a poco. (h.8)

Para los informantes clave, la reciprocidad que se da entre los adolescentes y sus amigos es también muy particular; así lo manifiestan:

-(...) El grupo de amigos ejerce una gran influencia para hacer o no algo; dígase competir en una carrera, empezar a fumar o tomar e incluso desobedecer a los padres. (i.c.7)

Tal y como es expresado por los adolescentes y los informantes clave, el joven, en cuanto a su grupo de pares, lo visualiza "como el inductor de seguridad, el árbitro de aceptación o rechazo y el indicador de lo permitido y lo prohibido, de lo aceptable y lo inaceptable". (Martínez, 2000: 220). La relación de amistad no siempre es negativa, los grupos les brindan a los adolescentes el afecto, la comprensión y la solidaridad que no consiguen con sus padres ni con otros adultos, por las relaciones conflictivas ya señaladas. No obstante, caen en las garras de las drogas porque representan una nueva exploración y alivio a sus vicisitudes cotidianas y, como ya se ha especificado hasta la saciedad, para estos muchachos y estas muchachas, el fenómeno de la adicción es parte de su entorno. 


\subsection{EL ENTORNO DE LA ESCUELA Y COLEGIO EN LOS ADOLESCENTES}

El aspecto más grave que se percibe en la educación primaria y secundaria en Costa Rica, una vez que se identifica el consumo de drogas ilícitas entre adolescentes, es que se crea un estigma en torno a la crisis y a los protagonistas, pero, a su vez, no se evidencian esfuerzos por alcanzar nuevas exploraciones de apoyo y tratamiento en la rehabilitación del joven o la joven sino que, por el contrario, se convierte en una lucha de poder en busca de culpables entre la escuela, el colegio y la familia, que en definitiva, no favorece la indagación tendiente a poner en práctica nuevas soluciones.

El abordaje del consumo problemático de drogas, es un fenómeno en el que participan muchos actores. Existe cierta tendencia a buscar culpables o responsables, pero la responsabilidad está muy compartida. Un guionista puede ser responsable de un tratamiento frívolo del consumo de drogas, el artista puede cantar una canción recibida de manera diferente por los adultos y menores, el programador se puede equivocar al elegir la hora de emisión de un espacio determinado, el periodista puede difundir información incorrecta o no contrastadas, los profesores pueden olvidarse de la educación a favor de los contenidos, $y$ de los padres y madres de utilizar la pequeña pantalla como guardería de sus hijos o como sedante de uso legal. (Martín. PDF/Adobe Acrobat - Versión en HTML. Http://www. Unad.org/).

Se ha comprobado dentro de algunas escuelas el tráfico de drogas ilícitas a veces introducidas en el entorno educativo por los adultos $y$, en ciertos casos, por los mismos jóvenes. Algunas apreciaciones de los adolescentes e informantes clave son las siguientes:

-(...) A lo interno de la escuela hay consumo e intercambio de sustancias y si no se da la intervención oportuna, evidentemente se convierte en un problema. (i.c.7)
-(...) Me sacaron del colegio; mis notas eran malas. (m.6)

-(...) Engañaba a los profesores para salirme del colegio y drogarme. (m.2)

-(...) Es impresionante, en el colegio, la entrada a sétimo cómo se relaciona con el inicio al consumo de drogas; ese cambio es muy drástico, y algunos no sobreviven sin marcas. (i.c.5)

Los adolescentes se convierten en infractores dentro de la escuela, por medio de un proceso, que cada vez se torna más complejo y de más difícil manejo; en consecuencia, la escuela termina sacándolo o el mismo estudiante deserta.

-(...) El colegio lo dejé; sacaba buenas notas cuando no consumía, pero luego inicié mi consumo de droga y ya no pude parar, ni siquiera iba al colegio; mentía en mi casa. (h.6)

-(...) Tuve muchos problemas en la escuela. Empecé el vandalismo; rayaba las paredes. (m.8)

-(...) En el colegio ya ni la directora me hacía caso; yo era un caso perdido. Me negaron la entrada al colegio (m.1)

- (...) yo iba a seguir estudiando, pero, por los problemas con la drogas, no seguí. (m.8)

- (...) Llegue hasta cuarto grado de la escuela. No me gustaba estudiar. (h.1)

Por esta razón, es posible articular el consumo de drogas en el adolescente, como una consecuencia del fracaso en el proceso de enseñanza, independientemente de calidad de la educación y de su funcionalidad con el acceso al trabajo o con la elevación de la calidad de vida de los que lograron concluir sus estudios de bachillerato. Los adolescentes fracasan en el proceso de instrucción $y$, por tanto, son excluidos por el mismo sistema, una situación que no resuelve la problemática del fenómeno de las 
drogas, pero que en definitiva introduce más al adolescente a la corriente de la adicción que al camino de la rehabilitación. Es responsabilidad del sistema de enseñanza en todos los niveles, la prevención de problemas psicosociales, los relacionados con el sistema escolar, la expulsión y el bajo nivel educativo de las familias, Consejo Nacional y PANI (2005).

En torno a la deserción escolar, el Ministerio de Educación apunta:

Entre las características de los estudiantes que desertan están: la mayoría cuen$\tan$ entre los 13 a 16 años de edad, trabajan en empleos de baja remuneración $y$ de poca estabilidad y en términos de factores familiares los desertores reciben poca ayuda de sus padres $y$ en sus hogares enfrentan problemas como la desintegración familiar, son además afectados por la drogadicción y los embarazos (Estado de la Nación, 2005: 26).

La deserción escolar es un problema determinante: constituye un detonador que actualmente está develando una crisis que el sistema educativo debe retomar para reflexionar y mejorar la calidad de la enseñanza, con un diseño de programas de enseñanza más atractivos para los jóvenes con metodologías innovadoras. También contar con una visión más integral de nuestros jóvenes y adolescentes, que vaya más allá de perfilarlos simplemente como un número en el aula de una escuela o colegio. La idea es verlos desde la perspectiva integral de un ser humano, con sus individualidades y sus capacidades dentro de un entorno familiar y comunal muy particular.

Sin embargo, para el combate del fenómeno de la drogadicción en la escuela, la CICAD/ OEA propone dentro de los principios de prevención escolar:

Cada escuela cuente con una política preventiva del consumo de drogas insertado en un proyecto educativo, defina un equipo responsable, cuente con profesores capacitados para abordar el tema, fije normas $y$ procedimientos para enfrentar los hechos del consumo, disponga de mecanismos de apoyo y derivación, defina programas de prevención universal, selectiva e indicada para todos los niveles educativos (CICAD/OEA, 2005:18).

El fundamento de los programas preventivos debe dirigirse según la OEA/CICAD (2005) a la promoción de estilos de vida por medio de determinados valores, actitudes y conocimientos apoyados en programas de fortalecimiento del adolescente. Estos basados en metodologías de habilidades para la vida, que permiten reforzar los factores psicosociales de los jóvenes, en una modalidad de educación participativa $y$ de construcción.

Otros datos que apuntan hacia el nivel de escolaridad del conjunto de adolescentes investigados se anotan a continuación: en el grupo de los ocho hombres, siete estudiaron la primaria incompleta y solamente uno llegó a la secundaria incompleta; del grupo de las ocho mujeres, solo una cursó la primaria incompleta $y$ las siete restantes avanzaron hasta la enseñanza secundaria incompleta.

Independientemente de los factores concretos que han determinado la interrupción de sus estudios, tanto hombres como mujeres, en el contexto costarricense se consideran y son valorados como personas que han fracasado en su proceso instructivo o, dicho desde la dimensión social, son excluidos del sistema que exige y demanda alumnos con estudios de bachillerato concluidos. Otra consideración digna de ser mencionada se relaciona con los distintos niveles de enseñanza en los que las mujeres y los hombres fracasan; en la primaria, lo hacen los hombres, mientras que en la secundaria son las mujeres. Esta relación invertida, en parte expresa la peculiaridad costarricense, dado que se trata de una sociedad que tempranamente alcanzó derechos igualitarios y un Estado social que respetó y amparó los derechos y calidad de vida ciudadana, $y$ posibilitó el acceso de la mujer a los procesos de instrucción, lo cual marca una distinción con respecto de otros países del área centroamericana.

Según el VI Informe del Estado de los derechos de la niñez y la adolescencia en Costa Rica (2008) el abandono temporal o permanente 
de los estudios, es una de las variables que más afecta la cobertura escolar, en ellas se encuentra la deserción, la cual se ha considerado como un proceso de exclusión del sistema. En la primaria, la deserción intra-anual en la educación formal es de un 4\%; sin embargo, el más bajo en el 2004 alcanzó un 3,3\%. En la secundaria las proporciones han decrecido más, en el 2004 el porcentaje fue de $11,6 \%$ que es una cifra que genera mucha preocupación.

De manera tal que el Sistema Educativo en nuestro país, debe delimitar estrategias que permitan disminuir más las cifras de deserción en la primaria y secundaria, con la finalidad de cumplir con el derecho a la educación para la población de niños y adolescentes, así como el establecimiento de mejores condiciones para evitar el abandono de los estudios y la posibilidad de mejorar las metodologías de enseñanza y el rendimiento académico.

\subsection{EL AMBIENTE DE LA COMUNIDAD Y LA INFLUENCIA EN EL CONSUMO DE DROGAS ILÍCITAS EN EL ADOLESCENTE}

La comunidad es el entorno donde conviven los adolescentes con sus familias; constituye un predominio, debido a que en ella convergen las costumbres, valores, principios y normas de arraigo de cada colectividad en particular, que, en su momento, posee una reciprocidad con los miembros que la componen.

La coexistencia de numerosos grupos sociales con disfuncionalidad en la dinámica familiar es correspondiente a la misma comunidad en forma recíproca: el entorno de las familias conflictivas influye sobre la comunidad $y$, a su vez, un colectivo social enfermo afecta a las familias en forma continua. La crisis de las drogas trae consigo otros conflictos sociales como coexistencia de la prostitución, enunciado más por las mujeres adolescentes que por los muchachos; asimismo, en ambos sexos se produce como resultado la delincuencia: roban, cometen delitos para mantenerse en el ciclo del consumo. Esto ocasiona, en definitiva, la desintegración social, que los sujetos de la investigación, adolescentes y cuidadores, respectivamente, expresan así:
- (...) Yo vivía en el centro de la droga; Hatillo 1, 15 de setiembre, San Sebastián son lugares donde aparece la droga por todo lado. Los policías no entran a estas comunidades; no hay una ley que impida el consumo ni la venta. (h.1)

-(...) Vivo en Puntarenas. Ahí está el mar $y$ el estero; los barcos entran y traen la droga; en mi barrio abunda la droga. La policía se vende; es parte de los grupos narcotraficantes; cuando hay operativos, ellos avisan a los narco para que estén preparados. (h.3)

- (...) Empecé consumiendo marihuana en mi casa; siempre anduve en la calle; luego pasé a la cocaína. Siempre estuve en la calle; me prostituía y robaba. Desciendo de una familia que trafica y consume droga. (m.8)

- (...) Sí, nosotros nacemos en un ambiente de drogas, prostitución; uno ve eso normal y empieza a practicarlo. (i.c.6)

El acceso a las drogas se facilita en los sectores donde viven los adolescentes. Su ambiente de pobreza implica la posesión de limitados recursos y lugares para que los jóvenes tengan un estilo de vida más sano: que practiquen la actividad física, el deporte, la recreación, entre otras actividades. En consecuencia, la droga se convierte en el primer elemento del medio ambiente comunal para estos jóvenes; se convive con las drogas y se facilita tanto el consumo como el tráfico de estas sustancias ilícitas.

- (...) La comunidad donde vivo es una área rural. Todo el mundo consumía; es un precario. Todas las personas consumen, hasta los niños, adultos y adultos mayores. (m.3)

- (...) La comunidad donde vivimos es un precario; es un ambiente malo. Todas las personas vienen de vender la droga $y$ también la consumen. (h.5)

- (...) Ambiente de la pobreza, de un estatus social bajo, en donde no hay lugares de 
recreación; no practican algún deporte. Este ambiente es la causa para consumir drogas. (i.c.4)

-(...) Si un ambiente es muy pesado o muy corrupto, hace un posible adolescente adicto o antisocial. (i.c.3)

Los adolescentes $y$ sus familias provienen de algunas de los zonas mencionadas por ellos, en sus relatos, rurales y urbanas; no hay diferenciación, pero sí existen lugares identificados a escala nacional en el tráfico y consumo de drogas como el cantón de Alajuelita y el distrito capitalino de Hatillo (provincia de San José) y otras zonas como Guápiles (cabecera del cantón de Pococí, provincia de Limón), y Jacó (en el cantón de Garabito, provincia de Puntarenas); ambos son ambientes portuarios, donde la habilidad para adquirir y trasegar la droga se facilita aun más a través de la vía marítima. Según el Instituto Costarricense sobre Drogas (2007) la ubicación del istmo centroamericano, facilita el transporte de las drogas ilícitas por varias vías como son: las terrestres, las aéreas y las marítimas. En la terrestre se transporta la cocaína, en la vía marítima se destaca el tráfico de importantes cargamentos de cocaína iguales o superiores a las dos toneladas de peso que se trasiegan en las costas centroamericanas con la finalidad de ocultarse y en la vía aérea se utilizan las avionetas que trasportan grandes cantidades de droga. El fenómeno del tráfico de la drogas ilícitas en nuestro país favorece la internalización y la comercialización, situación que hace más compleja la problemática.

Desde esta perspectiva, las condiciones de pobreza en algunas comunidades facilitan socialmente, la internacionalización del consumo y tráfico de las drogas ilícitas; el avance progresa sin ningún control del Estado y se convierte en un fenómeno que es parte de la subsistencia para muchas familias, aspecto que necesita ser retomado en el ámbito nacional e internacional. Actualmente, la drogodependencia desemboca directamente en colectividades que en el contexto local de cada país producen sufrimiento, desolación y muertes, lo cual, bajo un marco preventivo, de intervención y de voluntad política, puede evitarse.

\section{CONSIDERACIONES FINALES}

El Estado costarricense debe delimitar las políticas públicas en la dirección de la vigilancia y la exigencia en el cumplimiento de los derechos de la niñez, adolescencia y juventud en el país, con la finalidad de optimizar la toma de decisiones para mejorar la calidad de vida del consabido grupo poblacional en estudio.

La metodología cualitativa utilizada en esta investigación del fenómeno de las drogas, con la aplicación de la técnica del grupo focal, permitió la expresión de sentimientos y significados desde los adolescentes y las adolescentes, a partir de sus propias vivencias del fenómeno de la adicción a las drogas. Este aspecto favoreció la convivivencia, solidaridad y la cohesión grupal, lo que proporcionó la recolección de datos de la investigación para el análisis y la reflexión.

La adolescencia es un proceso de transformación que implica una mayor vulnerabilidad al cambio social por parte de los adolescentes, en la búsqueda constante de una identidad propia, situación que puede favorecer la adopción de conductas de riesgo, como es el consumo de drogas ilícitas; además se trata de una etapa en la que también desempeña un papel importante el medio social en el que se desenvuelven los jóvenes. En dicho entorno, los protagonistas vienen a ser los amigos a través de una relación más horizontal, debido a la imposibilidad de los padres para mantener relaciones armoniosas con sus hijos y una comunicación más fluida.

La globalización de los mercados y el consumismo en la época posmoderna construye una visión de mundo diferente a este grupo social de adolescentes, con cambios y consecuencias por las innovaciones particulares del contexto universal como son, el uso de la tecnología cada vez más sofisticada, el mundo de la moda, la música, todos, en múltiples ocasiones imitados por ellos. Estas experiencias conllevan aspectos ocultos, cargados de situaciones riesgosas, por ejemplo, el sexo sin protección, el uso y abuso de sustancias lícitas e ilícitas en este grupo poblacional son parte de esos mensajes subliminales. Tal andamiaje 
deja a los jóvenes en una posición de vulnerabilidad, pues prácticamente quedan inermes ante los distintos riesgos que deben afrontar en su entorno social.

La investigación reveló que los núcleos familiares a los que pertenecen estos jóvenes se hallan dirigidos por mujeres jefas de hogar, abandonadas por sus compañeros sentimentales, quienes no han asumido la responsabilidad paterna. Y, en consecuencia, ellas tienen que luchar con la pobreza en un colectivo social en el que se trafica y se consume la droga, situación que se percibe como una oportunidad para la subsistencia de la familia y representa para los adolescentes un círculo muy difícil de romper; y así lo han evidenciado en sus relatos.

La familia se ha considerado, social e históricamente, como un mecanismo fundamental en la vida del ser humano y de las sociedades; sin embargo, se evidencia, a través de los testimonios de los jóvenes entrevistados, que existe disfuncionalidad de la dinámica familiar, aspecto que se refleja en la pobreza, agresión, hogares constituidos por un solo miembro, mujeres jefas de hogar. Los citados factores constituyen una nebulosa de riesgo para los muchachos $y$ las muchachas, por cuanto los dejan a merced de la forma de pensar y los consejos de los amigos, quienes, con su "asesoría", en ciertas ocasiones empujan al adolescente hacia el camino de la drogadicción, como única salida a la encrucijada familiar y a su falta de adaptación socioeconómica y cultural.

El sistema educativo costarricense excluye al joven consumidor de drogas ilícitas del sistema de instrucción, debido a su bajo rendimiento escolar, así como a su comportamiento delictivo. Se trata de una política que más bien desemboca en la impotencia para que los jóvenes se alejen de las drogas, puedan recuperarse de su proceso de adicción y logren concluir sus proyectos inmediatos, como optimizar su calidad de vida y alcanzar el éxito social. Asimismo, evidencia la imposibilidad del sistema de enseñanza para abordar la crisis de la drogadicción en los adolescentes, para afrontarla y delimitar nuevas estrategias con el objeto de combatirla.

\section{REFERENCIAS BIBLIOGRÁFICAS}

Aigneren, M. "La técnica de recolección de información mediante los grupos focales". Revista electrónica 7. Biblioteca Virtual en Población. Centro Centroamericano de Población. 2009. En: <http:// huitoto. udea.edu.co/ ceo/>

Amara J. Desarrollo de la identidad. Asociación Mexicana de Pediatría. México: Graw-Hill M, editor, 2000.

Arenas, Ana y Villalobos, Sonia. "El maltrato infantil y adolescente". Documento de Trabajo. Patronato Nacional de la Infancia, San José, Costa Rica, 2008.

Arenas, Ana y Villalobos, Sonia. "El abuso sexual en niños y adolescentes". Documento de Trabajo. Patronato Nacional de la Infancia, Costa Rica, 2007.

Bejarano, Julio. "Evaluación de un programa escolar en Costa Rica basado en habilidades para la vida". Adicciones 17 (1). Sección Latinoamericana Sociedad Científica Española de Estudios sobre el Alcohol, el Alcoholismo y otras Toxicomanías. 2005: 70-80.

Castellano, B. "El adolescente y su entorno: Sociedad, familia y amigos". Revista Pediatría Integral IX (1). España, 2005: 41-46.

Díaz, Josefina. "Identidad, adolescencia y cultura: jóvenes secundarios en un contexto regional". Investigación Temática. Revista Mexicana de Investigación Educativa 11 (29), abril-junio. 2006: 431-457.

Dulanto, Enrique. El adolescente. Asociación Mexicana de Pediatría, Ac. México: Editorial Mc Graw-Hill Interamericana, 2000. 
Estado de la Nación. Programa de Estado de la Nación en Desarrollo Humano Sostenible. Estado de la Educación Costarricense. Programa de Estado de la Nación. Consejo Nacional de Rectores. San José, 2005.

Fishman, H.Charles. Tratamiento de adolescentes con problemas. Un enfoque de terapia familiar. Buenos Aires: Editorial Paidós, 1989.

Hesse, M.; Vanderplasschen, W.; Rapp, RC.; Broekaert, E.; Fridell, M. Manejo de casos para personas con trastornos por uso de sustancias (Revisión Cochrane traducida). En: La Biblioteca Cochrane Plus, 2008. Número 2. Oxford: Update Software Ltd. En: <http://www.update-software.com> (Traducida de The Cochrane Library, 2008 Issue 2. Chichester, UK: John Wiley \& Sons, Ltd.).

Hernández. "Construir una identidad. Vida juvenil y estudio en el Colegio de Ciencias y Humanidades Sur". Revista Mexicana de Investigación Educativa 11 (29), México, abril-junio 2006: 459-481.

Hidalgo, Vicario y J. Júdez Gutiérrez. "Adolescencia de alto riesgo. Consumo de drogas y conductas delictivas". Pediatria Integral XI (10). España. 2007: 895-910.

Instituto Costarricense sobre Drogas. Plan Nacional sobre Drogas 2008-2012. Presidencia de la República. San José, Costa Rica, 2007.

Instituto sobre Alcoholismo $y$ Fármacodependencia. La juventud y las drogas: Encuesta Nacional, sobre percepciones y consumo en población de educación secundaria. San José, Costa Rica, 2006.

Horkheimer, Max. Autoridad y familia. Buenos Aires: Editorial Paidós, 2001. pp. 207M.
Lagarde, Marcela. "Autoestima y género". Cuadernos Inacabados. México. 2000: $1-18$.

Leveton, Eva (1984). El adolescente en crisis, su apoyo en terapia familiar. México: Editorial Pax, 1987.

Mejía, Julio. "Sobre la investigación cualitativa. Nuevos Conceptos y campos de desarrollo". Revista investigaciones Sociales 13. Lima, Perú. 2004: 277-299.

Martínez, José Luis (1998). El adolescente y sus Pares. Asociación Mexicana de Pediatría, AC. México: Editorial Mc Graw-Hill Interamericana, 2000.

Martín, Javier. Medios de comunicación y el consumo de drogas de menores. [disponible en formato de archivo: PDF/ Adobe Acrobat-Versión en HTML] En: $<$ www.unad.org/>

Mendizábal José y Anzures Beatriz. "La familia y el adolescente". Revista Médica 62 (3), Hospital General de México, jul.-sep. 1999: 191-197.

Organización de Estados Americanos. Lineamientos Hemisféricos de la CICAD en prevención escolar. Comisión Interamericana para el control del abuso de Drogas, Washington D. C., 2005.

Patronato Nacional de la Infancia. Política de niñez y adolescencia. Consejo Nacional de la Niñez y Adolescencia, San José, Costa Rica. 2005.

Rojas, V Milton J. Factores de riesgo y protectores identificados en adolescentes consumidores de sustancias psicoactivas. Revisión y análisis del estado actual. Cedro, San José s/f.

Solum, Donas. "Protección, riesgo y vulnerabilidad". Revista. Adolesc. 
Latinoam. 1 (4), dic. 1999: 222-230. ISSN 1414-7130.

Universidad de Costa Rica, Unicef. Estado de los Derechos de la Niñez y la Adolescencia en Costa Rica. A los diez años del Código de Niñez y la Adolescencia. San José, Costa Rica, 2008. 
